\title{
Noninvasive Assessment of Crohn's Disease Intestinal Lesions with ${ }^{18} \mathrm{~F}-\mathrm{FDG}$ PET/CT
}

\author{
Edouard Louis ${ }^{1}$, Geoffrey Ancion ${ }^{2}$, Arnaud Colard ${ }^{1}$, Veronique Spote ${ }^{3}$, Jacques Belaiche ${ }^{1}$, and Roland Hustinx ${ }^{2}$ \\ ${ }^{1}$ Department of Gastroenterology, CHU of Liège, University of Liège, Liège, Belgium; ${ }^{2}$ Department of Nuclear Medicine, CHU of Liège, \\ University of Liège, Liège, Belgium; and ${ }^{3}$ Department of Medical Imaging, CHU of Liège, University of Liège, Liège, Belgium
}

\begin{abstract}
Pilot studies have shown good sensitivity and specificity for ${ }^{18} \mathrm{~F}-$ FDG PET in detecting gastrointestinal lesions of Crohn's disease. The combination of ${ }^{18} \mathrm{~F}-\mathrm{FDG}$ PET with CT may further improve the localization and characterization of lesions with increased ${ }^{18} \mathrm{~F}-\mathrm{FDG}$ uptake. Our aim was to assess the use of ${ }^{18} \mathrm{~F}-\mathrm{FDG}$ $\mathrm{PET} / \mathrm{CT}$ in evaluating the activity and location of Crohn's disease along the gastrointestinal tract. Methods: After giving informed consent, 22 patients with Crohn's disease were prospectively studied. They underwent ${ }^{18} \mathrm{~F}-\mathrm{FDG}$ PET/CT, followed by ileocolonoscopy within $1 \mathrm{wk}$ (mean, $2 \mathrm{~d}$ ). The Crohn's disease activity index (CDAl) was calculated, and serum C-reactive protein (CRP) and fecal calprotectin were measured before endoscopy. The Crohn's disease endoscopy index of severity (CDEIS) was calculated during endoscopy. The global CDEIS score and endoscopic subscores for various ileocolonic segments were used for analysis. Results: Globally, 95 intestinal and colonic segments in 22 patients were analyzed. ${ }^{18} \mathrm{~F}$-FDG PET/CT detected 35 of 48 endoscopically affected segments (sensitivity for the detection of endoscopic lesions, $72.9 \%$ ). The sensitivity of ${ }^{18} \mathrm{~F}-\mathrm{FDG}$ PET/CT for the detection of severe endoscopic lesions (deep ulcers and strictures) was 100\% (14/14). The global PET/CT score significantly correlated with CDEIS $(r=0.51 ; 95 \%$ confidence interval [Cl], 0.09-0.77; $P=0.017)$, CDAl $(r=0.58 ; 95 \% \mathrm{Cl}$, $0.17-0.80 ; P=0.005)$, and CRP ( $r=0.56$; 95\% Cl, 0.19-0.81; $P=0.007$ ). Conclusion: ${ }^{18} \mathrm{~F}-\mathrm{FDG} \mathrm{PET} / \mathrm{CT}$ was globally well correlated to the clinical, endoscopic, and biologic activity of Crohn's disease. Above all, this technique had a good sensitivity for the detection of intestinal and colonic segments with moderate to severe mucosal lesions. The potential impact of this promising tool on the global management of patients with Crohn's disease should be further evaluated in prospective studies.
\end{abstract}

Key Words: Crohn's disease; positron emission tomography; Crohn's disease endoscopic index of severity; CT scanner; Crohn's disease activity index

J Nucl Med 2007; 48:1053-1059

DOI: 10.2967/jnumed.107.040436

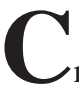

rohn's disease is a chronic inflammatory disease of the gastrointestinal tract. Its clinical course is characterized by

Received Feb. 2, 2007; revision accepted Mar. 27, 2007.

For correspondence or reprints contact: Edouard Louis, Service of Gastroenterology, CHU de Liège, 4000 Liège, Belgium.

E-mail: Edouard.louis@ulg.ac.be

COPYRIGHT @ 2007 by the Society of Nuclear Medicine, Inc. a succession of periods of clinical relapse and remission. The goal of treatment is to maintain a normal quality of life for the patient by sustaining remission and avoiding complications. With this goal, 2 different situations may be problematic for the clinician: patients with symptoms suggesting active disease but with biologic markers within the normal range, and patients with clinically inactive disease but who may silently evolve toward stricturing or fistulizing complications. In the first situation, a differential diagnosis must be made between truly active inflammatory disease, associated irritable bowel symptoms, and aftereffects from previous surgery. Blood inflammatory markers such as C-reactive protein (CRP) serum concentration, or stool inflammatory markers such as fecal calprotectin, may be helpful in such situations but are imperfectly correlated to inflammatory intestinal lesions (1-3). In the second situation, often after a clinically effective treatment, it may be tempting to try simply to prolong the obtained clinical remission. However, recent data have clearly shown that complications of Crohn's disease inexorably develop with time $(4,5)$. Immunosuppressors such as azathioprine and, more recently, biologic treatments such as infliximab have shown a potential for obtaining mucosal healing in Crohn's disease $(6,7)$. This mucosal healing has been associated with a higher sustained quality of life, lower rate of hospitalization, and lower need for surgery (8). Hence, not only a sustained clinical remission but also a control of intestinal lesions has become the target of new treatment strategies in Crohn's disease. Endoscopic evaluation has been considered the gold standard to assess intestinal lesions, and mucosal healing has been considered the Holy Grail for new treatments. However, ileocolonoscopy is invasive, unpleasant for the patient, sometimes incomplete because of unreachable segments, and able to assess only mucosal lesions although the disease may sometimes affect deeper parts of the bowel wall.

${ }^{18} \mathrm{~F}-\mathrm{FDG} \mathrm{PET} / \mathrm{CT}$ is a noninvasive technique that allows quantifying and precisely localizing ${ }^{18} \mathrm{~F}-\mathrm{FDG}$ uptake in the entire body. ${ }^{18} \mathrm{~F}-\mathrm{FDG}$ uptake is caused by increased local metabolic activity. Such increased uptake has been described not only in neoplastic lesions but also in inflammatory lesions $(9,10)$. In this condition, uptake has been correlated with local stimulation of tumor necrosis factor 
(11) and with monocyte priming and activation (12). A variable uptake may be observed in the bowel, especially the cecum (13), and has limited the use of PET in inflammatory bowel diseases. The advent of combined PET and CT devices may lead to significant improvements in the interpretation of the bowel areas. Indeed, the combination of PET and CT criteria greatly reduces the number of falsepositive findings in the gastrointestinal tract (14). In a patient with an established diagnosis of Crohn's disease, for whom a therapeutic decision has to be made or the effect of a treatment has to be evaluated on the basis of an objective assessment of the extent and severity of the lesions, ${ }^{18} \mathrm{~F}-$ FDG PET/CT may be of particularly great value. Our aim was to evaluate the performance of ${ }^{18} \mathrm{~F}$-FDG PET/CT in assessing the endoscopic index of activity of Crohn's disease and to correlate it with clinical score and biologic markers of disease activity.

\section{MATERIALS AND METHODS}

\section{Patients and Study Design}

Twenty-two consecutive Crohn's disease patients undergoing ileocolonoscopy for medical reasons were included between January 2006 and July 2006. Inclusion criteria were an age greater than $18 \mathrm{y}$ and the need for endoscopic evaluation of clinically or biologically active disease. All patients gave informed consent for the study protocol. This protocol was accepted by the Liège University Hospital Ethics Committee. Within 1 wk of the endoscopic procedure, the patients underwent ${ }^{18} \mathrm{~F}$-FDG PET/CT. The Crohn's disease activity index (CDAI) (15) was calculated, and CRP serum concentration and fecal calprotectin were measured the day of the ${ }^{18} \mathrm{~F}$-FDG PET/CT examination, a mean of $2 \mathrm{~d}$ before endoscopy. CRP was measured using a routine procedure. Fecal calprotectin was measured using a commercial ELISA device (Novatec).

\section{Ileocolonoscopy}

Ileocolonoscopies were performed after macrogol preparation, by senior endoscopists experienced in the field of Crohn's disease and unaware of the results of other evaluations. For endoscopic assessment, 5 segments per patient were considered: terminal ileum, right colon, transverse colon, left colon (including sigmoid), and rectum. The Crohn's disease endoscopy index of severity (CDEIS) was calculated (16). A subscore of the CDEIS was also extracted for each segment explored, including the presence of deep ulcers, the presence of superficial ulcers, the estimated surface area of affected mucosa, and the estimated surface area of ulcerated mucosa. The endoscopic lesions in each segment were also classified into 4 categories according to the maximal lesion in the segment, with 0 being no lesion; 1 being nonulcerated lesions (e.g., erythema or pseudopolyp), erosions, or aphthoid ulcers; 2 being larger superficial ulcers; 3 being deep ulcers; and 4 being stricture. Type 1 lesions were considered mild; type 2 were considered moderate; and types 3 and 4 , severe. Photographs and 2 biopsies per segment in areas of maximal inflammation were taken from the various segments explored. Histology was assessed by a senior pathologist specialized in digestive diseases.

\section{${ }^{18}$ F-FDG PET/CT Procedure and Scores}

Procedure. All PET/CT studies were performed using a Gemini Dual system (Philips). Patients fasted for $6 \mathrm{~h}$ before receiving $3.7 \mathrm{MBq}$ of ${ }^{18} \mathrm{~F}-\mathrm{FDG}$ per kilogram $(0.1 \mathrm{mCi} / \mathrm{kg})$ through an indwelling catheter. Blood glucose levels were measured before the injection and were lower than $130 \mathrm{mg} / \mathrm{dL}$ in all cases. The uptake period was $60 \mathrm{~min}$, during which the patients were asked to drink a total of 6 glasses of water $(1,500 \mathrm{~mL}$ total $)$ as a negative contrast agent for CT. The acquisition procedure was as follows: First, a spiral CT acquisition was performed from the base of the lungs to the inguinal folds $(2 \times 2.5-\mathrm{mm}$ slice thickness; pitch, 1.5 ; table feed, $15 \mathrm{~mm} / \mathrm{s} ; 120 \mathrm{kV} ; 50 \mathrm{mAs}$ ). The increment for reconstructing the images was $1.6 \mathrm{~mm}$. The patients were freely breathing, and no intravenous contrast agent was administered. Immediately after the CT study and without moving the patient, the PET emission scan was started. It consisted of 5-6 bed positions of $3 \mathrm{~min}$ each. PET images were reconstructed using an iterative algorithm (3-dimensional row-action maximum-likelihood algorithm) and were fully corrected for decay, scatter, random events, and attenuation. The complete procedure took $20 \mathrm{~min}$ on average. The radiation was estimated to be approximately $10 \mathrm{mSv}(0.1 \mathrm{mSv}$ for the CT scout view, $4.5 \mathrm{mSv}$ for the CT, and $5.7 \mathrm{mSv}$ for the ${ }^{18}$ F-FDG study).

PET/CT Image Analysis. All studies were analyzed by $2 \mathrm{nu}-$ clear medicine physicians and a radiologist who were masked to the study. All conclusions were reached by consensus.

The images were first analyzed using a semiquantitative visual scale taking the liver activity as a reference. A score of 0 corresponded to an activity lower than the liver, 1 was equal to the liver, and 2 was more active than the liver. Each segment of the digestive tract was systematically analyzed, with the help of the CT images for a precise localization (stomach, small bowel, distal ileum, ascending colon, transverse colon, descending and sigmoid colon, rectum, and anus).

For the CT analysis, each segment was analyzed and received a score of 0 when found to be normal and a score of 1 when any structural anomaly was noted. The parietal thickness was considered abnormal when thicker than $2 \mathrm{~mm}$ in the small bowel and thicker than $3 \mathrm{~mm}$ in the large bowel.

Quantitative Metabolic Analysis. Large $\left(40 \mathrm{~cm}^{2}\right)$ regions of interest were placed on the right lobe of the liver. Smaller $(0.96$ $\mathrm{cm}^{2}$ ) regions of interest were placed on all segments that received a score of 1 or 2 according to the visual analysis, on the transverse slice displaying the highest activity. The maximum-pixel-value standardized uptake value (SUV), normalized for body weight, according to previously published data, was used (SUVmax) (17). The SUV is a semiquantitative assessment of ${ }^{18} \mathrm{~F}$-FDG uptake and is fairly reproducible, provided the acquisition parameters are controlled (17). SUV is used in whole-body oncologic PET but has been shown inadequate for evaluating brain tumors, which are better assessed using lesion-to-normal-tissue (either cortex or white matter) activity ratios. Because no method is fully validated for inflammatory bowel disease, we decided to evaluate both SUV and lesion-to-normal-tissue activity ratio. Because the liver has been shown to have low within-patient variability (17), we chose this organ as the reference for normal tissue activity. We therefore calculated the ratios of each positive-segment SUVmax over the liver SUVmax (RSUV). All analyses were performed using both the SUVmax and the ratios (RSUV), but because the correlations with endoscopic, biologic and clinical scores were better with the latter, mainly those are reported in this article. 
Global PET/CT Score. A global PET/CT score was calculated by summing the SUVmax for every studied segment and dividing by the number of segments studied. Two scores were calculated: The first, called the ileocolonoscopy PET/CT score, was based on the segments (1-5) studied at ileocolonoscopy to correlate with CDEIS, and the second, called the global PET/CT score, was based on 7 segments representing the rectum, left colon, transverse colon, right colon, terminal ileum, small bowel, and stomach.

\section{Statistical Analyses}

Various endoscopic, PET, and CT scores were attributed to each segment explored and were compared or correlated. The scores for endoscopy reflected either the mean severity (segment CDEIS) or the maximum severity of the segment (lesion types 0-4). For PET and CT, the maximum severity was considered. The sensitivity and specificity for the detection of segments with endoscopic lesions by PET/CT were calculated. The values of SUVmax according to the type of endoscopic lesion (types 0-4) were compared using the Kruskal-Wallis test. The proportions of severe endoscopic lesions (types 3 and 4) according to PET/CT appearance (no ${ }^{18} \mathrm{FDG}$ uptake or bowel wall thickening, isolated increased ${ }^{18} \mathrm{~F}$-FDG uptake, or both increased ${ }^{18} \mathrm{~F}$-FDG uptake and bowel wall thickening) were compared by the $\chi^{2}$ test $(3 \times 2$ contingency table). The correlation between SUVmax and segment CDEIS subscore was calculated by the Spearman test. A logistic regression was performed to assess the value of SUVmax and bowel wall thickening in the detection of either severe (types 3 and 4) or moderate to severe (types 2, 3, and 4) endoscopic lesions in each individual segment. The receiver operating characteristic curve was then used to determine a threshold value for SUVmax for the detection of severe lesions.

Correlations between the 2 global PET/CT scores, CDEIS, CDAI, CRP, and fecal calprotectin were calculated by the Spearman test. Ileocolonoscopy PET/CT scores, CRP, CDAI, and fecal calprotectin were compared according to a CDEIS threshold value of 6 , representing a validated threshold for clinically relevant lesions at endoscopy (18).

A $P$ value of less than 0.05 was considered statistically significant.

\section{RESULTS}

The characteristics of the patients are presented in Table 1. A full ileocolonoscopy was possible in 17 cases; in 5 cases, the endoscopic exploration was stopped in the transverse colon ( 2 cases), the left colon (1 case), or the rectum ( 2 cases) because of strictures. Using ${ }^{18} \mathrm{~F}-\mathrm{FDG}$ PET/ $\mathrm{CT}$, it was possible to analyze all the segments explored by endoscopy. It was also possible to analyze segments not reached during endoscopy ( 5 ileal segments, 5 right colons, 3 transverse colons, 2 left colons), as well as the rest of the small bowel and the stomach for all patients.

\section{Analysis of Segments Explored by ${ }^{18}$ F-FDG PET/CT and Endoscopy}

Overall, 95 intestinal and colonic segments were explored by endoscopy and ${ }^{18}$ F-FDG PET/CT. Of these 95 segments, 47 showed no endoscopic lesion, 16 showed type 1 lesions, 18 showed type 2 lesions, 7 showed type 3 lesions, and 7 showed type 4 lesions. Fifty-six segments showed increased ${ }^{18}$ F-FDG uptake, and 39 showed bowel
TABLE 1

Characteristics of the 22 Patients

\begin{tabular}{|c|c|}
\hline Characteristic & Value \\
\hline Male patients $(n)$ & 7 \\
\hline \multicolumn{2}{|l|}{ Age $(y)$} \\
\hline Median & 32.5 \\
\hline Range & $20-69$ \\
\hline \multicolumn{2}{|l|}{ Disease duration (mo) } \\
\hline Median & 60 \\
\hline Range & $5-384$ \\
\hline \multicolumn{2}{|l|}{ Disease location } \\
\hline Colonic & 11 \\
\hline Ileal & 2 \\
\hline Ileocolonic & 9 \\
\hline \multicolumn{2}{|l|}{ Treatment } \\
\hline Mesalazine & 5 \\
\hline Antibiotics & 1 \\
\hline Steroids & 6 \\
\hline Immunosuppressors & 10 \\
\hline Anti-tumor necrosis factor & 8 \\
\hline \multicolumn{2}{|l|}{ CDAI } \\
\hline Median & 178 \\
\hline Range & $54-331$ \\
\hline \multicolumn{2}{|l|}{ CDEIS } \\
\hline Median & 6.5 \\
\hline Range & $0-28$ \\
\hline \multicolumn{2}{|l|}{ CRP (mg/L) } \\
\hline Median & 17.4 \\
\hline Range & $1.1-78.1$ \\
\hline \multicolumn{2}{|l|}{ Fecal calprotectin $(\mu \mathrm{g} / \mathrm{mL})$} \\
\hline Median & 535 \\
\hline Range & $8.4-1,250$ \\
\hline
\end{tabular}

wall thickening on CT. All segments with bowel wall thickening showed increased ${ }^{18} \mathrm{~F}-\mathrm{FDG}$ uptake. Globally, ${ }^{18} \mathrm{~F}-$ FDG PET/CT detected 35 of 48 endoscopically affected segments (example in Fig. 1A) (sensitivity for the detection of endoscopic lesions, 72.9\%). Among the segments abnormal on ${ }^{18} \mathrm{~F}$-FDG PET/CT, 35 of 56 showed endoscopic lesions, and among segments without endoscopic lesions, 26 of 47 were also ${ }^{18}$ F-FDG PET/CT-negative (specificity for the detection of endoscopic lesions, 55.3\%). When considering only segments showing both increased ${ }^{18} \mathrm{~F}-\mathrm{FDG}$ uptake and bowel wall thickening on CT, the sensitivity for the detection of endoscopic lesions was 54.2\% (26/48), whereas the specificity increased to $72.3 \%$ (34/47). The ${ }^{18} \mathrm{~F}$-FDG PET/CT results according to the various types of lesions are shown in Table 2.

The sensitivity of ${ }^{18} \mathrm{~F}-\mathrm{FDG}$ PET/CT for the detection of severe endoscopic lesions (deep ulcers and strictures) was $100 \%$, and the sensitivity to detect moderate to severe lesions (types 2, 3, and 4 lesions: superficial ulcers, deep ulcers, and strictures) was $84.4 \%$.

A significant correlation was found between RSUV and CDEIS segment subscores $(r=0.46$ [95\% confidence interval, $0.28-0.61] ; P<0.0001)$. The intensity of ${ }^{18} \mathrm{~F}-$ FDG uptake significantly increased according to the severity of endoscopic lesions $(P<0.0001)$ (Fig. 2). Severe (types 3 and 4) endoscopic lesions were more frequent in 

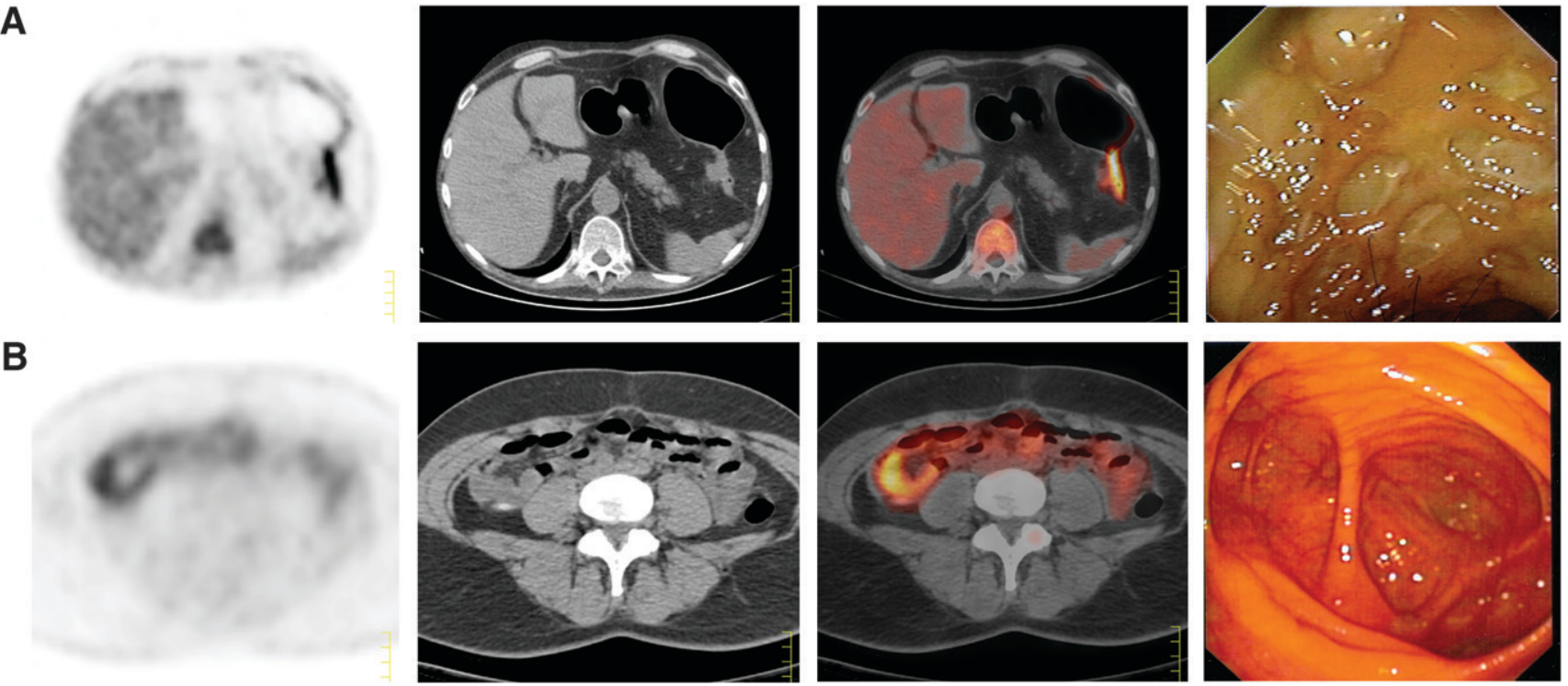

FIGURE 1. From left to right, examples of PET, CT, PET/CT, and corresponding endoscopic appearance. (A) Deep ulcers with cobblestones in left colon, appearing as thickened segment with prominent increase of ${ }^{18} \mathrm{~F}-\mathrm{FDG}$ uptake on PET/CT. (B) No endoscopic lesion in cecum, contrasting with thickening of bowel wall and increased uptake of FDG on PET/CT.

segments with bowel wall thickening: The proportion of type 3 and 4 lesions in segments with no ${ }^{18}$ F-FDG uptake, isolated ${ }^{18} \mathrm{~F}$-FDG uptake, and both increased ${ }^{18} \mathrm{~F}-\mathrm{FDG}$ uptake and bowel wall thickening was $0 \%, 16.6 \%$, and $30 \%$, respectively $(P=0.0006)$. A logistic regression showed that the segment RSUV was significantly associated with the presence of severe (types 3 and 4) endoscopic lesions $(P=0.0002)$, whereas the bowel wall thickening was not independently associated with the presence of severe lesions. At a threshold of 1.47 for RSUV, the sensitivity for the diagnosis of severe endoscopic lesions (deep ulcers and strictures) in the considered segment was $100 \%$, and the specificity $67.1 \%$. The receiver operating characteristic curve for the detection of severe endoscopic lesions is shown in Figure 3. At this threshold, the sensitivity for the detection of any endoscopically affected segment was 31 of 48 (64.6\%), and the specificity was 76.6\%. Among the 21 segments showing increased ${ }^{18} \mathrm{~F}-\mathrm{FDG}$ uptake but no endoscopic lesion, 10 presented a thickening of the bowel wall, 5 presented both thickening of the bowel wall and active inflammation on histologic examination, and 1 presented isolated active mucosal inflammation on histologic examination, suggesting a local inflammatory process affecting mucosa or deeper layers of the bowel wall not detected at endoscopy in 16 of 21 cases (76.2\%) (example in Fig. 1B).

\section{Correlations Between Global PET/CT Score and Other Markers}

The correlations between the global PET/CT score and clinical, endoscopic, or biologic scores and markers of activity are shown in Table 3.

Global PET/CT score, CDAI, and CRP, but not fecal calprotectin, were significantly different in patients with or without clinically relevant endoscopic lesions (Table 4).

\section{DISCUSSION}

To our knowledge, this study was the first on the correlation between ${ }^{18} \mathrm{~F}$-FDG PET/CT, endoscopic evaluation, and clinical and biologic scores of activity in Crohn's disease. A couple of ${ }^{18} \mathrm{~F}-\mathrm{FDG}$ PET studies have already been reported in Crohn's disease (19-21). They showed the good performance of this technique in assessing intestinal lesions and disease activity. The specificity of the technique was, however, difficult to evaluate because ${ }^{18} \mathrm{~F}-\mathrm{FDG}$ uptake without endoscopic lesions was difficult to interpret and was usually considered a false-positive result of ${ }^{18} \mathrm{~F}-\mathrm{FDG}$

TABLE 2

PET/CT Results According to Endoscopic Lesions Detected in the 95 Segments Explored by the 2 Techniques

\begin{tabular}{|c|c|c|c|c|c|c|}
\hline \multirow[b]{2}{*}{ Endoscopic lesion } & \multirow[b]{2}{*}{ No. of PET-positive segments } & \multicolumn{2}{|c|}{ SUV } & \multicolumn{2}{|c|}{ RSUV } & \multirow[b]{2}{*}{ No. of thickened segments at CT } \\
\hline & & Median & Range & Median & Range & \\
\hline None & $21 / 47$ (44.7\%) & 0 & $0-14.72$ & 0 & $0-5.66$ & 13/47 (27.7\%) \\
\hline Type 1 & $8 / 16(50 \%)$ & 1.22 & $0-4.9$ & 0.39 & $0-1.96$ & $4 / 16(25 \%)$ \\
\hline Type 2 & $13 / 18(72.2 \%)$ & 4.27 & $0-11.69$ & 1.65 & $0-4.5$ & $10 / 18(55.6 \%)$ \\
\hline Type 3 & $7 / 7$ (100\%) & 5.62 & $3.19-14.10$ & 2.81 & $1.47-5.77$ & 6/7 (85.7\%) \\
\hline Type 4 & 7/7 (100\%) & 3.95 & $3.36-7.31$ & 2.04 & $1.68-4.06$ & 6/7 (85.7\%) \\
\hline
\end{tabular}




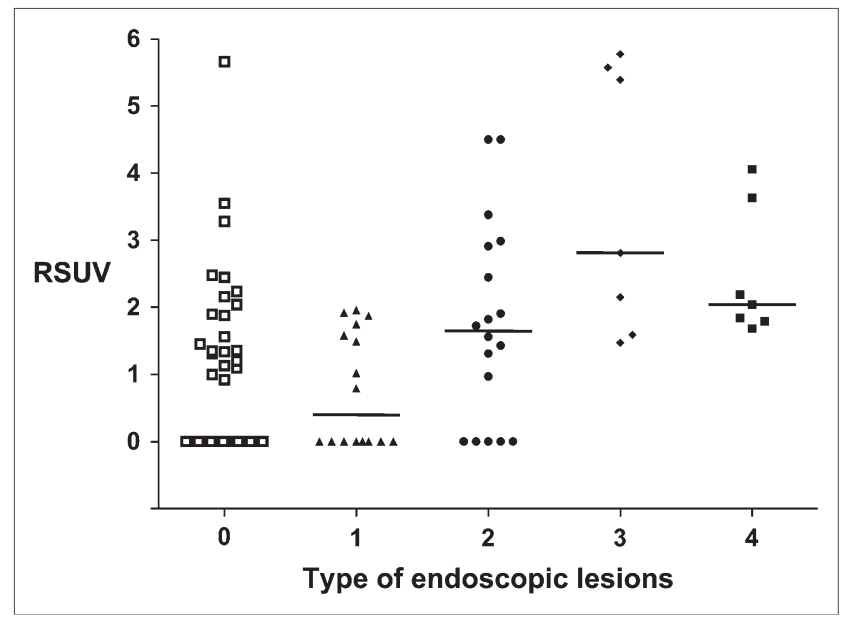

FIGURE 2. RSUV for each of 95 intestinal segments explored according to type of endoscopic lesion. RSUV (ratio of SUV in bowel segments over liver SUV) was significantly different in the 5 types of lesions $(0=$ no lesion; 1 = aphthoid ulcers, erythema, and pseudopolyps; 2 = superficial ulcers; 3 = deep ulcers and cobblestones; 4 = stricture). Bars indicate medians (median for type 0 lesion is 0$)(P<0.0001)$.

PET. Coupling with CT allows a more precise locating of ${ }^{18} \mathrm{~F}$-FDG uptake, a more precise assessment of the various intestinal and colonic segments, and a more adequate evaluation of positive segments. Several other nuclear medicine techniques have been proposed in this setting, in particular

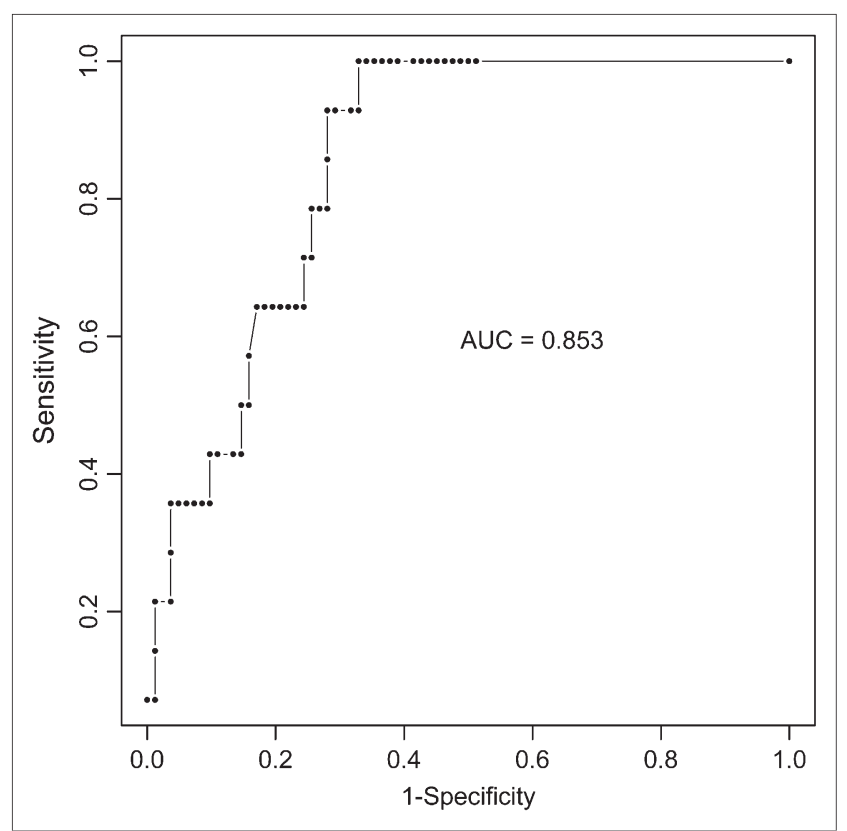

FIGURE 3. Receiver operating characteristic curve of RSUV for detection of severe endoscopic lesions (types 3 and 4: deep ulcers and strictures). We deliberately selected a threshold with maximum sensitivity to allow detection of the vast majority of severe lesions. In our dataset, a threshold of 1.47 of RSUV gave a sensitivity of $100 \%$ for detection of severe lesions and a specificity of $67.1 \%$ for detection of any endoscopic lesion.
99mTc-hexamethylpropyleneamine oxime leukocytes, with reasonably high diagnostic accuracy $(22,23)$. Such an approach, however, did not fully find its place in the clinical diagnostic algorithms and also presents several methodologic drawbacks, such as a quite cumbersome labeling procedure and the risks associated with handling blood derivatives.

The most important results of our study with ${ }^{18} \mathrm{~F}-\mathrm{FDG}$ PET/CT probably come from the analysis of individual intestinal segments. The ${ }^{18} \mathrm{~F}-\mathrm{FDG}$ PET/CT aspect was studied in 95 ileal and colonic segments of 22 patients. The ${ }^{18} \mathrm{~F}-$ FDG uptake measured by RSUV in each segment correlated well and significantly with the CDEIS subscore and with the type and severity of endoscopic lesions for the considered segment. The sensitivity of ${ }^{18} \mathrm{~F}-\mathrm{FDG}$ PET/CT for the detection of endoscopic lesions was globally reasonably good, at around $70 \%$. Importantly, the only undetected segments with ${ }^{18} \mathrm{~F}$-FDG PET/CT harbored only mild to moderate endoscopic lesions, including erythema or superficial ulcers. All segments with deep ulcers, cobblestones, or strictures were detected with ${ }^{18} \mathrm{~F}-\mathrm{FDG}$ PET/CT, giving rise to a sensitivity of $100 \%$ for severe lesions. Globally, the association of bowel wall thickening on CT with abnormal ${ }^{18} \mathrm{~F}-\mathrm{FDG}$ uptake increased the specificity for the detection of endoscopic lesions. However, an RSUV threshold could be calculated that kept a maximum sensitivity (100\%) for the detection of severe lesions while increasing the specificity for the detection of any endoscopic lesion (mild to severe) up to $76.6 \%$, which was superior to the specificity of the association of increased ${ }^{18} \mathrm{~F}$-FDG uptake without threshold and bowel wall thickening on CT. Furthermore, according to logistic regression, the bowel wall thickening on CT did not add to the predictive value of the intensity of ${ }^{18} \mathrm{~F}-\mathrm{FDG}$ uptake (assessed by RSUV) for the presence of severe endoscopic lesions in a considered segment. However, the CT scanner still allowed us to precisely locate the lesions and gave some morphologic information, such as the presence of a significant stricture. In clinical practice, the disappearance of at least moderate to severe lesions may be relevant for the future management of the patient and therapeutic decisions. The performance of other noninvasive techniques for the evaluation of the intestine and colon, essentially entero-CT and hydro-MRI, has also been reported as good. However, these techniques have been evaluated mainly in the assessment of small-bowel Crohn's disease and more rarely for colonic Crohn's disease. Furthermore, most of these studies lack a precise description of the nature and severity of endoscopic lesions (24). Therefore, an unambiguous comparison with our results for ${ }^{18} \mathrm{~F}$-FDG PET/CT in the detection of various types of endoscopic lesions is difficult. In a recent study evaluating MRI colonography in the assessment of inflammatory bowel disease, sensitivity for the detection of inflamed ileal and colonic segments at endoscopy in Crohn's disease was globally around 30\% (25). The detection of mild inflammation was particularly difficult, with only 2 
TABLE 3

Correlations between Global PET/CT Score and Clinical, Endoscopic, or Biologic Scores and Markers

\begin{tabular}{|c|c|c|c|c|c|c|}
\hline Parameter & CDAI & CDEIS & $\begin{array}{c}\text { Fecal } \\
\text { calprotectin }\end{array}$ & CRP & $\begin{array}{c}\text { Global ileocolonic } \\
\text { PET/CT score }\end{array}$ & $\begin{array}{c}\text { Global } \\
\text { PET/CT score }\end{array}$ \\
\hline CDAI & & $\begin{array}{c}0.52(0.11-0.78) \\
P=0.014\end{array}$ & -; $P=0.23$ & $\begin{array}{c}0.48(0.06-0.76) \\
P=0.024\end{array}$ & $\begin{array}{c}0.45(0.02-0.74) \\
P=0.036\end{array}$ & $\begin{array}{c}0.58(0.19-0.81) \\
P=0.005\end{array}$ \\
\hline CDEIS & & & -; $P=0.07$ & $\begin{array}{c}0.66(0.09-0.77) \\
P=0.0008\end{array}$ & $\begin{array}{c}0.51(0.09-0.77) \\
P=0.017\end{array}$ & -;P = 0.11 \\
\hline Fecal calprotectin & & & & $\begin{array}{c}0.54(0.06-0.81) \\
P=0.028\end{array}$ & $-; P=0.52$ & -;P $=0.54$ \\
\hline CRP & & & & & $\begin{array}{c}0.49(0.07-0.76) \\
P=0.022\end{array}$ & $\begin{array}{c}0.56(0.17-0.80) \\
P=0.007\end{array}$ \\
\hline
\end{tabular}

Data are Spearman correlation coefficient, with 95\% confidence interval in parentheses. Global ileocolonic PET/CT score has been calculated on the basis of 5 intestinal segments from terminal ileum to rectum, whereas global PET/CT score has been calculated on the basis of 7 gastrointestinal tract segments from stomach to rectum.

(7.4\%) of 27 segments detected, as compared with $50 \%-$ $72 \%$ for comparable lesions with ${ }^{18} \mathrm{~F}-\mathrm{FDG}$ PET/CT in the present study. More severe lesions were better detected (8/ 9 ), but the detection was not superior to that of ${ }^{18} \mathrm{~F}-\mathrm{FDG}$ PET/CT as described in the present study. A better sensitivity for the detection of endoscopic lesions with ${ }^{18} \mathrm{~F}-\mathrm{FDG}$ PET than with hydro-MRI had also already been found in the first prospective study evaluating ${ }^{18} \mathrm{~F}$-FDG PET in Crohn's disease (20). The specificity for the detection of inflamed segments at endoscopy seemed somewhat higher with MRI colonography that with the results we found for ${ }^{18}$ F-FDG PET/CT (25). However, such was not the case in a previous study comparing these 2 techniques (20), and as discussed later, these apparently false-positive segments with ${ }^{18} \mathrm{~F}-\mathrm{FDG}$ PET/CT must be interpreted with caution because bowel wall thickening on CT or inflammation on histologic examination was present in most cases. Ultimately, these techniques should be prospectively compared. A potential advantage over ${ }^{18} \mathrm{~F}$-FDG PET/CT is that MRI or CT better characterizes the extent of the lesions and potential extramural complications. A further advantage of MRI is the absence of irradiation, although irradiation with ${ }^{18} \mathrm{~F}-\mathrm{FDG}$ PET/CT is globally similar to that with an enteroscanner.

Some segments showed increased ${ }^{18}$ F-FDG uptake although harboring no endoscopic lesions. These segments would have been considered false-positive using ${ }^{18} \mathrm{~F}-\mathrm{FDG}$
PET alone, and we considered them as such for the calculation of the capacity of detection of endoscopic lesions in the present study. However, the coupled analysis of PET and CT revealed that these positive PET areas were indeed located in the bowel wall and that most showed bowel wall thickening suggesting an ongoing pathologic process deeper in the bowel wall. This bowel wall thickening was also associated in a few cases with active histologic inflammation. Such results had already been observed in previous studies evaluating ${ }^{18} \mathrm{~F}-\mathrm{FDG}$ PET in Crohn's disease (19). An elegant way to clarify this observation would be to look at the bowel wall using endoscopic ultrasonography to better characterize the transmural pathologic process. This ability to detect active intestinal disease beyond the mucosa would also be another asset for ${ }^{18} \mathrm{~F}-\mathrm{FDG}$ PET/CT, because complications of Crohn's disease such as strictures may probably develop without many mucosal lesions being visible at endoscopy, and because extraenteric inflammation is increasingly recognized as an important feature in Crohn's disease (26).

We have also shown a significant correlation between a global PET/CT score based on the mean RSUV for the various intestinal segments studied and CDAI, CDEIS, and CRP. Globally, this correlation reflects the good performance of ${ }^{18} \mathrm{~F}-\mathrm{FDG}$ PET/CT in assessing disease activity in Crohn's disease patients, at the clinical, biologic, and endoscopic levels. The global correlation with CDEIS was

TABLE 4

CDAI, CRP, Fecal Calprotectin, and Global PET/CT Score According to a Predefined CDEIS Threshold (18)

\begin{tabular}{lccc}
\hline \multicolumn{1}{c}{ Parameter } & CDEIS $\geq 6$ & CDEIS $<6$ & $P$ \\
\hline CDAl & $260(109-331)$ & $135(54-210)$ & 0.007 \\
CRP $(\mathrm{mg} / \mathrm{L})$ & $38.5(1.4-78.1)$ & $7.7(1.1-21.3)$ & 0.0015 \\
Fecal calprotectin $(\mu \mathrm{g} / \mathrm{mL})$ & $765.5(100.3-1,250)$ & $400.6(10-1,250)$ & 0.17 \\
Global ileocolonic PET/CT score & $2.13(0.38-4.52)$ & $0.66(0.38-2.4)$ & 0.014
\end{tabular}

Data are median, with range in parentheses. 
rather good and was particularly better than for fecal calprotectin but was slightly lower than for CRP, and obviously CRP would thus be an easier and more straightforward marker for global mucosal disease activity in Crohn's disease. Interestingly, like CRP and CDAI but unlike fecal calprotectin, the global PET/CT score was significantly different between patients with or without clinically relevant mucosal lesions, defined according to a threshold previously validated in a GETAID study (18). Here again, however, CRP seems to be a more straightforward marker. When the global PET/CT score was extended to all abdominal gastrointestinal tract segments, including nonterminal small bowel and stomach, the correlation with CDAI was better than when the global score was calculated on the basis of ileocolonic segments. This global gastrointestinal PET/CT score correlated better with CDAI than with CDEIS or CRP, suggesting an impact of pan-digestive tract inflammation on the clinical activity of the disease.

\section{CONCLUSION}

This prospective study comparing ${ }^{18} \mathrm{~F}$-FDG PET/CT with endoscopic, clinical, and biologic scores of activity in Crohn's disease has shown a good correlation between scores derived from ${ }^{18} \mathrm{~F}$-FDG PET/CT and the clinical, endoscopic, and biologic activity of the disease. The most interesting asset of ${ }^{18} \mathrm{~F}-\mathrm{FDG}$ PET/CT could be its ability to detect and localize almost all gastrointestinal segments with moderate to severe lesions in the bowel wall and a significant proportion of the segments with only mild lesions, allowing a noninvasive evaluation of the ongoing pathologic process in the gastrointestinal tract. However, the significant patient irradiation linked to the technique will impede frequent use. Furthermore, the significance of increased ${ }^{18} \mathrm{~F}-\mathrm{FDG}$ uptake without a visible endoscopic lesion in some cases remains to be clarified. Finally, the impact of this technique on the management and treatment of patients with Crohn's disease should be further evaluated.

\section{ACKNOWLEDGMENTS}

This work was supported by a FIRS grant from the CHU of Liège and by an unrestricted research grant from AstraZeneca Belgium. Edouard Louis is Senior Research Associate at the FNRS of Belgium.

\section{REFERENCES}

1. Cellier C, Sahmoud T, Froguel E, et al. Correlations between clinical activity, endoscopic severity, and biological parameters in colonic or ileocolonic Crohn's disease: a prospective multicentre study of 121 cases. Gut. 1994;35:231-235.

2. Roseth AG, Aadland E, Grzyb K. Normalization of faecal calprotectin: a predictor of mucosal healing in patients with inflammatory bowel disease. Scand J Gastroenterol. 2004;39:1017-1020.
3. Costa F, Mumolo MG, Ceccarelli L, et al. Calprotectin is a stronger predictive marker of relapse in ulcerative colitis than in Crohn's disease. Gut. 2005;54: 364-368.

4. Louis E, Collard A, Oger AF, et al. Behaviour of Crohn's disease according to the Vienna Classification: changing pattern over the course of the disease. Gut. 2001;49:777-782.

5. Cosnes J, Cattan S, Blain A, et al. Long-term evolution of disease behaviour of Crohn's disease. Inflamm Bowel Dis. 2002;8:244-250.

6. D'Haens G, Geboes K, Rutgeerts P. Endoscopic and histologic healing of Crohn's (ileo)-colitis with azathioprine. Gastrointest Endosc. 1999;50:667-671.

7. Rutgeerts P, Diamond RH, Bala M, et al. Scheduled maintenance treatment with infliximab is superior to episodic treatment for the healing of mucosal ulceration associated with Crohn's disease. Gastrointest Endosc. 2006;63:433-442.

8. Rutgeerts P, Feagan BG, Lichtenstein GR, et al. Comparison of scheduled and episodic treatment strategies of infliximab in Crohn's disease. Gastroenterology. 2004;126:402-413.

9. Yamada S, Kubota K, Kubota R, Ido T, Tamahashi N. High accumulation of fluorine-18-fluorodeoxyglucose in turpentine-induced inflammatory tissue. $\mathrm{J} \mathrm{Nucl}$ Med. 1995;36:1301-1306.

10. Love C, Tomas MB, Tronco GG, Palestro CJ. FDG PET of infection and inflammation. Radiographics. 2005;25:1357-1368.

11. Jones HA, Cadwallader KA, White JF, Uddin M, Peters AM, Chilvers ER. Dissociation between respiratory burst activity and deoxyglucose uptake in human neutrophil granulocytes: implications for interpretation of ${ }^{18} \mathrm{~F}$-FDG PET images. J Nucl Med. 2002;43:652-657.

12. Paik JY, Lee KH, Choe YS, Choi Y, Kim BT. Augmented ${ }^{18}$ F-FDG uptake in activated monocytes occurs during the priming process and involves tyrosine kinases and protein kinase C. J Nucl Med. 2004;45:124-128.

13. Cook GJ, Maisey MN, Fogelman I. Normal variants, artefacts and interpretative pitfalls in PET imaging with 18-fluoro-2-deoxyglucose and carbon-11 methionine. Eur J Nucl Med. 1999;26:1363-1378.

14. Kamel EM, Thumshirn M, Truninger K, et al. Significance of incidental ${ }^{18} \mathrm{~F}-$ FDG accumulations in the gastrointestinal tract in PET/CT: correlation with endoscopic and histopathologic results. J Nucl Med. 2004;45:1804-1810.

15. Best W, Becktel JM, Singleton JW, et al. Development of a Crohn's disease activity index. Gastroenterology. 1976;70:439-444.

16. Mary JY, Modigliani R. Development and validation of an endoscopic index of the severity for Crohn's disease: a prospective multicentre study. Groupe d'Etudes Therapeutiques des Affections Inflammatoires du Tube Digestif (GETAID). Gut. 1989;30:983-989.

17. Paquet N, Albert A, Foidart J, Hustinx R. Within-patient variability of ${ }^{18}$ F-FDG: standardized uptake values in normal tissues. J Nucl Med. 2004;45:784-788.

18. Mary J, Lemann M, Colombel JF, et al. Endoscopic remission and response in Crohn's disease: an objective definition using the CDEIS [abstract]. Gut 2005; 54(suppl VII):A50.

19. Bicik I, Bauerfeind P, Breitbach T, von Schulthess GK, Fried M. Inflammatory bowel disease activity measured by positron-emission tomography [letter]. Lancet. 1997;350:262.

20. Neurath MF, Vehling D, Schunk K, et al. Noninvasive assessment of Crohn's disease activity: a comparison of ${ }^{18} \mathrm{~F}$-fluorodeoxyglucose positron emission tomography, hydromagnetic resonance imaging, and granulocyte scintigraphy with labeled antibodies. Am J Gastroenterol. 2002;97:1978-1985.

21. Lemberg DA, Issenman RM, Cawdron R, et al. Positron emission tomography in the investigation of pediatric inflammatory bowel disease. Inflamm Bowel Dis. 2005;11:733-738.

22. Molnar T, Papos M, Gyulai C, et al. Clinical value of technetium-99m-HMPAOlabeled leukocyte scintigraphy and spiral computed tomography in active Crohn's disease. Am J Gastroenterol. 2001;96:1517-1521.

23. Charron M, Di Lorenzo C, Kocoshis SCT. ${ }^{99 \mathrm{~m}} \mathrm{Tc}-\mathrm{WBC}$ vs colonoscopy in the evaluation of inflammation and complications of inflammatory bowel diseases. J Gastroenterol. 2002;37:23-28.

24. Mackalski BA, Bernstein CN. New diagnostic imaging tools for inflammatory bowel disease. Gut. 2006;55:733-741.

25. Schreyer AG, Rath HC, Kikinis R, et al. Comparison of magnetic resonance imaging colonography with conventional colonoscopy for the assessment of intestinal inflammation in patients with inflammatory bowel disease: a feasibility study. Gut. 2005;54:250-256.

26. Colombel JF, Solem CA, Sandborn WJ, et al. Quantitative measurement and visual assessment of ileal Crohn's disease activity by computed tomography enterography: correlation with endoscopic severity and C reactive protein. Gut. 2006;55:1561-1567. 